\title{
Coffee and black tea consumption and breast cancer mortality in a cohort of Swedish women
}

\author{
HR Harris*, ${ }^{*, 2}$, L Bergkvist ${ }^{3}$ and A Wolk' \\ 'Division of Nutritional Epidemiology, The National Institute for Environmental Medicine, Karolinska Institutet, PO Box 21 0, Stockholm I7I 77, Sweden; \\ ${ }^{2}$ Obstetrics and Gynecology Epidemiology Center, Brigham and Women's Hospital, Harvard Medical School, 221 Longwood Avenue, Boston, \\ MA 02115, USA; ${ }^{3}$ Department of Surgery and Centre for Clinical Research, Central Hospital, Västerås 721 89, Sweden
}

BACKGROUND: Coffee and black tea contain a mixture of compounds that have the potential to influence breast cancer risk and survival. However, epidemiologic data on the relation between coffee and black tea consumption and breast cancer survival are sparse. METHODS: We investigated the association between coffee and black tea consumption and survival among 3243 women with invasive breast cancer in the Swedish Mammography Cohort. Intake was estimated using a food frequency questionnaire. Cox proportional hazard models were used to calculate hazard ratios (HRs) and $95 \%$ confidence intervals $(95 \% \mathrm{Cls}$ ).

RESULTS: From 1987 to 2010 there were 394 breast cancer-specific deaths and 973 total deaths. Coffee and black tea were not associated with breast cancer-specific or overall mortality. Women consuming $4+$ cups of coffee per day had a covariate and clinical characteristics-adjusted HR $(95 \% \mathrm{Cl})$ of death from breast cancer of I.I $4\left(0.7 \mathrm{I}-1.83 ; p_{\text {trend }}=0.8 \mathrm{I}\right)$ compared with those consuming $<$ I cup per day. Women consuming $2+$ cups of black tea per day had a covariate and clinical characteristics-adjusted HR (95\% Cl) of death from breast cancer of I.02 (0.67-1.55; $\left.p_{\text {trend }}=0.94\right)$ compared with non-tea drinkers. Caffeine was also not associated with breast cancer-specific (HR for top to bottom quartile $=1.06 ; 95 \% \mathrm{Cl}=0.79-1.44 ;$ trend $=0.7 \mathrm{I}$ ) or overall mortality.

CONCLUSION: Our findings suggest that coffee, black tea, and caffeine consumption before breast cancer diagnosis do not influence breast cancer-specific and overall survival.

British Journal of Cancer (2012) 1 07, 874-878. doi:I0.1038/bjc.20 I2.337 www.bjcancer.com

Published online 26 July 2012

(C) 2012 Cancer Research UK

Keywords: breast cancer; epidemiology; coffee; tea; caffeine; survival

Coffee and black tea contain a mixture of compounds that have the potential to influence breast cancer risk and survival. Coffee may influence risk and progression through the inhibition of DNA methylation (Lee and Zhu, 2006), influences on tumour differentiation (Pozner et al, 1986), or alterations in sex hormone levels (Ferrini and Barrett-Connor, 1996; Nagata et al, 1998). Black tea has also been shown to alter sex hormone levels (Kuruto-Niwa et al, 2000; Wu et al, 2005), and the flavonoids it contains may have antioxidant effects (Trevisanato and Kim, 2000). Caffeine is found in both coffee and black tea, and in rodents has been shown to increase mammary cell differentiation (VanderPloeg et al, 1992) and decrease tumour incidence (Petrek et al, 1985). Conversely, caffeine has also been associated with increased mammary tumours in animal models (Welsch et al, 1983).

Coffee consumption and breast cancer risk have been extensively studied with conflicting results (Ishitani et al, 2008; Bissonauth et al, 2009; Larsson et al, 2009a; Tang et al, 2009; Bhoo Pathy et al, 2010; Boggs et al, 2010; Nilsson et al, 2010; Fagherazzi et al, 2011; Gierach et al, 2011; Li et al, 2011). However, to our knowledge, only one observational study has examined coffee consumption and survival following breast cancer diagnosis (Sugiyama et al, 2010), and no studies have examined black tea or caffeine. In this study, we investigated whether pre-diagnosis

*Correspondence: Dr HR Harris; E-mail: holly.harris@ki.se Received 4 May 2012; revised 3 July 2012; accepted 6 July 2012; published online 26 July 2012 coffee, black tea, and caffeine intake were associated with breast cancer survival among women diagnosed with invasive breast cancer in the population-based Swedish Mammography Cohort (SMC). We also examined whether the association between coffee, black tea, or caffeine and survival differed by hormone receptor status, disease stage at diagnosis, or smoking status.

\section{MATERIALS AND METHODS}

\section{Study population}

This study included 3243 participants in the SMC with invasive breast cancer diagnosed from 1987 to 2010. Recruitment and characteristics of this cohort have been previously described (Wolk et al, 2006). In brief, the SMC is a population-based cohort of 66651 women born between 1914 and 1948 that were recruited between 1987 and 1990 in Västmanland and Uppsala counties in central Sweden. Participants completed a baseline questionnaire with questions regarding diet, reproductive, and other factors. In 1997, a second questionnaire was extended to include dietary supplements, physical activity, and smoking status, and was sent to participants who were still alive and residing in the study area; $39227(70 \%)$ women returned this questionnaire. The study was approved by the ethics committee at the Karolinska Institutet.

Histologically confirmed incident invasive breast cancer cases were ascertained by linkage of the study cohort with the Swedish Cancer Registry. This registry has been estimated to 
provide almost $100 \%$ complete case ascertainment (Mattsson and Wallgren, 1984). Oestrogen receptor (ER) and progesterone receptor (PR) status and other clinical characteristics were obtained by reviewing pathology laboratory works logs from Uppsala University Hospital and by linkage with the clinical database at the Regional Oncology Centre in Uppsala. Oestrogen and PR status, menopausal status at diagnosis, tumour size, grade, lymph node involvement, and type of treatment were available for $\sim 72 \%$ of the cases. More detailed information on the evaluation of hormone receptor status in this cohort has been described previously (Larsson et al, 2009b).

\section{Dietary assessment}

Diet was assessed using a 67-item food frequency questionnaire (FFQ) at baseline and a 96-item FFQ in 1997. Participants were asked how often, on average, they had consumed coffee and tea during the previous 6 months (1987) or year (1997). Eight responses were possible ranging from never or seldom to four or more times per day. Decaffeinated coffee and tea were rarely consumed in Sweden during the study period. As the majority of tea consumed during the study period was black tea, we refer to tea as black tea throughout the manuscript. Caffeine intake was calculated by summing the caffeine content of coffee and tea multiplied by the amount of consumption using values obtained from the Swedish National Food Administration Database. The FFQ has been previously validated among SMC participants with correlation coefficients between the questionnaire and four 1-week dietary records of 0.6 for coffee and 0.8 for tea (A Wolk, unpublished data, 1992).

\section{Outcome assessment}

Date of death was identified through linkage to the Swedish National Death Registry at Statistics Sweden. Cause of death was determined by International Classification of Diseases (ICD) codes (ICD9 and ICD10) through linkage to the Cause of Death Registry at the National Bureau of Health and Welfare.

\section{Statistical analysis}

Cox proportional hazard models with time since diagnosis in months as the time scale were used to calculate hazard ratios (HRs) and $95 \%$ confidence intervals $(95 \%$ CIs) for death from breast cancer. Participants contributed person-time from the date of breast cancer diagnosis until death from breast cancer (primary endpoint), death from another cause, or end of follow-up on 16 October 2010. Secondary analyses were conducted with death from any cause as the endpoint. Coffee consumption was categorised as $<1$ (reference), $1,2-3$ and $\geqslant 4$ cups per day. Black tea consumption was categorised as non-drinker (reference), $<1,1$ cup per day, and $\geqslant 2$ cups per day. Caffeine intake was categorised in quartiles with the lowest quartile as the reference group. Total caloric intake and age at diagnosis were included in all models.

Education level, marital status, menopausal status at diagnosis, body mass index (BMI), alcohol intake, and calendar year of diagnosis were included in all multivariable models. Additional multivariable models were adjusted for the following clinical characteristics: stage, grade, radiation treatment, and chemotherapy/hormonal therapy. Additional adjustment for the clinical covariates tumour size and number of positive lymph nodes did not further alter the effect estimates; thus they were not included in the final model. We also adjusted for physical activity (Holmes et al, 2005) and smoking status among women who completed the 1997 questionnaire. Coffee and black tea were included simultaneously in all models. Tests for linear trend were performed by assigning the median value of each category to each participant in that group.
We examined whether the association between coffee, black tea, and caffeine consumption and breast cancer survival differed by hormone receptor status, disease stage at diagnosis, or smoking status with a likelihood ratio test comparing the model with the cross-product term between each exposure variable and each potential effect modifier to the model with main effects only. All tests of statistical significance were two-sided, and statistical analyses were performed using SAS Version 9.2 (SAS Institute Inc., Cary, NC, USA).

\section{RESULTS}

During 28676 person-years of follow-up contributed by 3243 breast cancer cases, there were 973 deaths with 394 deaths from breast cancer. Ninety-six percentage of participants reported any coffee consumption and seventy-three percentage reported any black tea consumption. Among those consuming coffee or black tea, the median consumption among the drinkers was 2.5 and 1.0 cups per day, respectively. Median caffeine consumption among all participants was $326 \mathrm{mg}$ per day. Women consuming $\geqslant 4$ cups of coffee per day tended to be younger, had a lower mean BMI, were less often nulliparous, consumed more alcohol, consumed less black tea, and were more likely to smoke than those in lower categories of coffee intake (Table 1).

No association was observed between coffee consumption and breast cancer-specific or overall mortality. Women consuming $\geqslant 4$ cups of coffee per day had a covariate-adjusted HR (95\% CI) of death from breast cancer of $1.01(0.64-1.61)$ compared with those consuming $<1$ cup per day $\left(p_{\text {trend }}=0.99\right)$. Adjustment for clinical characteristics (breast cancer stage and grade) and treatment did not materially alter the results $\left(1.14(0.71-1.83) ; p_{\text {trend }}=0.81\right)$. Results were similar when death from any cause was the outcome (Table 2). When coffee consumption was dichotomised, comparing women consuming $\geqslant 1$ cup per day to those consuming $<1$ cup per day there was no association with breast cancer-specific $(\mathrm{HR}=1.17 ; 95 \% \mathrm{CI} 0.80-1.72)$ or overall mortality $(\mathrm{HR}=1.02$; 95\% CI 0.81-1.29). Caffeine intake was not associated with breast cancer-specific (HR for top (median intake $488 \mathrm{mg}$ per day) to bottom (median intake $155 \mathrm{mg}$ per day) quartile $=0.99 ; 95 \%$ $\mathrm{CI}=0.74-1.33 ; p_{\text {trend }}=0.93$ ) or overall mortality (HR for top to bottom quartile $\left.=1.07 ; 95 \% \mathrm{CI}=0.88-1.30 ; p_{\text {trend }}=0.67\right)$.

Black tea consumption was nonsignificantly inversely associated with breast cancer-specific and overall mortality. Compared with women consuming no black tea those consuming $\geqslant 2$ cups per day had a covariate-adjusted HR $(95 \% \mathrm{CI})$ of death from breast cancer of $0.87\left(0.58-1.32 ; p_{\text {trend }}=0.58\right)$ and 0.84 for total deaths $\left(0.64-1.09 ; p_{\text {trend }}=0.39\right)$. Adjustment for clinical characteristics and treatment attenuated the results (HR for breast-cancer deaths $=1.02 ; 95 \% \mathrm{CI}=0.67-1.55 ; p_{\text {trend }}=0.94$ and total deaths $=$ $0.94 ; 95 \% \mathrm{CI}=0.72-1.23 ; p_{\text {trend }}=0.88$ ) (Table 3$)$. When women with any black tea consumption were compared with those with no black tea consumption, no association was observed with breast cancer-specific ( $\mathrm{HR}=0.98 ; 95 \%$ CI $0.78-1.22)$ or overall mortality $(\mathrm{HR}=0.94 ; 95 \%$ CI $0.82-1.09)$

The associations between coffee, black tea, and caffeine intake and mortality did not vary by hormone receptor status (Supplementary Tables 1 and 2), disease stage at diagnosis, or smoking (results not shown) (all $P>0.05$ ). In addition, adjustment for ER/ PR status in the covariate and clinical characteristics model did not materially change the results. In a sensitivity analysis that excluded women with stage IV breast cancer, results were not materially different. We also adjusted for physical activity and smoking status in the subset of women who completed the 1997 questionnaire and saw no material change in the effect estimates.

We examined dietary change following breast cancer diagnosis among the 691 breast cancer cases who were diagnosed with breast cancer from 1987 to 1996 and completed a FFQ in 1997 after their 
Table I Characteristics of 3234 women with invasive breast cancer in the Swedish Mammography Cohort by coffee intake ${ }^{a}$

\begin{tabular}{|c|c|c|c|c|}
\hline & \multicolumn{4}{|c|}{$\begin{array}{l}\text { Coffee intake } \\
\text { (cups per day) }\end{array}$} \\
\hline & $<\mathbf{I}$ & $\mathbf{I}$ & $2-3$ & $\geqslant 4$ \\
\hline Median coffee intake (g per day) & 12 & 177 & 443 & 768 \\
\hline Age at enrollment (years) & 52.4 & 56.1 & 53.1 & 48.5 \\
\hline Age at diagnosis (years) & 65.6 & 68.6 & 65.8 & 62.1 \\
\hline Post-secondary education (\%) & 16.8 & 14.3 & 13.0 & 14.4 \\
\hline Married (\%) & 65.3 & 68.6 & 71.7 & 71.3 \\
\hline Body mass index $\left(\mathrm{kg} \mathrm{m}^{-2}\right)$ & 25.2 & 25.2 & 24.9 & 24.7 \\
\hline Height $(\mathrm{cm})$ & 165.3 & 164.9 & 164.5 & 165.1 \\
\hline Age at menarche (years) & 13.2 & 13.3 & 13.2 & 13.1 \\
\hline Nulliparous (\%) & 14.2 & 17.5 & 11.7 & 11.2 \\
\hline Age at first birth among parous women (years) & 24.6 & 24.9 & 24.8 & 23.7 \\
\hline Number of children & 2.3 & 2.3 & 2.2 & 2.4 \\
\hline Family history of breast cancer (\%) & 10.6 & 13.9 & 10.7 & 9.5 \\
\hline Ever use of oral contraceptives (\%) & 58.7 & 48.6 & 55.8 & 63.7 \\
\hline Ever use of postmenopausal hormones (\%) & 46.8 & 51.3 & 45.8 & 47.1 \\
\hline Postmenopausal at diagnosis (\%) & 91.2 & 94.6 & 91.2 & 89.2 \\
\hline Alcohol intake (grams per day) & 2.2 & 2.4 & 2.9 & 3.4 \\
\hline Current smoker (\%) & 5.9 & 5.8 & 11.8 & 24.8 \\
\hline Past smoker $(\%)^{b}$ & 32.5 & 31.0 & 33.6 & 43.3 \\
\hline Tea intake (cups per day) & 1.3 & 0.9 & 0.5 & 0.3 \\
\hline Caffeine intake (mg per day) & 105.5 & 175.1 & 338.7 & 523.1 \\
\hline Total energy intake (kcal per day) & 1532 & 1498 & 1587 & 1688 \\
\hline \multicolumn{5}{|l|}{ Disease stage $(\%)^{\mathrm{c}, \mathrm{d}}$} \\
\hline Stage I & 51.7 & 50.2 & 52.5 & 54.1 \\
\hline Stage II & 42.9 & 42.2 & 40.0 & 39.7 \\
\hline Stage III/IV & 3.9 & 6.9 & 5.5 & 5.2 \\
\hline \multicolumn{5}{|l|}{ Treatment (\%) ${ }^{\mathrm{d}, \mathrm{e}}$} \\
\hline Radiation & 56.6 & 48.2 & 52.3 & 56.6 \\
\hline Chemotherapy & 21.2 & 12.3 & 13.1 & 17.2 \\
\hline Hormonal & 33.6 & 33.9 & 32.1 & 32.1 \\
\hline Oestrogen receptor positive (\%) ${ }^{\mathrm{d}}$ & 79.7 & 83.0 & 81.4 & 81.7 \\
\hline Progesterone receptor positive (\%) ${ }^{d}$ & 67.2 & 70.4 & 66.5 & 65.4 \\
\hline \multicolumn{5}{|l|}{ Tumour size (\%) ${ }^{d}$} \\
\hline$<2 \mathrm{~cm}$ & 66.0 & 59.5 & 60.9 & 63.7 \\
\hline $2-4 \mathrm{~cm}$ & 28.1 & 33.2 & 33.3 & 29.6 \\
\hline$>4 \mathrm{~cm}$ & 5.9 & 7.2 & 5.7 & 6.6 \\
\hline \multicolumn{5}{|l|}{ Number of positive-lymph nodes (\%) ${ }^{d}$} \\
\hline None & 57.6 & 63.8 & 65.6 & 65.3 \\
\hline $1-3$ & 27.3 & 25.8 & 22.9 & 23.2 \\
\hline$\geqslant 4$ & |5.1 & 10.5 & 11.6 & 11.5 \\
\hline
\end{tabular}

${ }^{a}$ Data represent mean unless otherwise indicated. ${ }^{b}$ Smoking status was only available from women $(n=2110)$ who completed the 1997 questionnaire. 'Percentages may not equal 100 owing to missing values. Information on clinical characteristics was available for $\sim 72 \%$ of the participants. ${ }^{~}$ Greater than $100 \%$ because some breast cancer patients received more than one treatment.

breast cancer diagnosis. Over $90 \%$ remained in the same or adjacent category of coffee or black tea intake following their breast cancer diagnosis. However, numbers were too small to examine the association between post-diagnosis coffee or black tea intake and survival in this group.

\section{DISCUSSION}

In this prospective study of Swedish women with breast cancer, we observed no association between coffee, primarily black tea, or caffeine intake and mortality following breast cancer diagnosis. This lack of association was consistent across hormone receptor subtypes, stage of diagnosis, and among both smokers and nonsmokers.
The epidemiologic data regarding the relation between coffee, tea, and caffeine and breast cancer survival is limited. Stocks compared age-adjusted death rates and annual consumption of tea and coffee in 20 countries and reported a positive association between tea and breast cancer death rates but no association with coffee (Stocks, 1970). To our knowledge, no observational studies have examined the association between tea or caffeine consumption and breast cancer survival, and only one study has examined the association with coffee consumption. Sugiyama et al (2010) examined coffee consumption and mortality due to all-cause, cardiovascular disease (CVD), and cancer in a prospective cohort study in Japan. They observed an inverse association between coffee consumption and overall and CVD mortality, but no association with total cancer mortality or breast cancer-specific mortality (HR $(95 \% \mathrm{CI})$ for breast cancerspecific mortality for 1 cup per day $v s$ none $=1.54(0.34-6.93)$ ); however, the breast cancer-specific results were based on only 19 deaths. Coffee intake was much lower among this population than in Sweden, which has one of the highest consumption rates in the world. Despite the different ranges of coffee consumption between the two study populations, we also did not observe a significant association between coffee intake and breast cancer mortality.

In the SMC, we have previously reported no association between coffee consumption and breast cancer risk and an increased risk of overall breast cancer as well as an increased risk for ER $+/ \mathrm{PR}+$ tumours with black tea consumption (Larsson et al, 2009a). The positive association between black tea consumption and hormone receptor-positive breast cancer risk may indicate a role of sex steroid hormones as in vitro studies have demonstrated that tea catechins exhibit oestrogenic activity in low concentrations (Kuruto-Niwa et al, 2000). The lack of association observed between black tea consumption and mortality suggests that consumption of black tea may influence breast cancer incidence and survival through different mechanisms.

A limitation of our study was for the majority of our participants we only had a pre-diagnosis assessment of diet and thus had limited power to examine diet post diagnosis during the follow-up period. Among a cohort of breast cancer patients in the United Kingdom, small but statistically significant changes in pre- to postdiagnosis intake of coffee (pre-diagnosis mean 1.05 servings per day and post-diagnosis mean 0.96 servings per day) and tea (pre-diagnosis mean 1.55 servings per day and post-diagnosis mean 1.61 servings per day) were observed (Velentzis et al, 2011). However, $90 \%$ of our participants who completed an FFQ post diagnosis remained in the same or adjacent category of coffee and black tea consumption following diagnosis. In addition, other dietary or lifestyle factors may have changed following breast cancer diagnosis. Studies have shown that younger women are most likely to report these changes and the average age at breast cancer diagnosis in our cohort was 65.1 years (Salminen and Lagstrom, 2000; Maunsell et al, 2002). Coffee and black tea consumption were assessed with a self-administered FFQ, which is subject to some measurement error and could have resulted in attenuation of the true association. However, in this cohort the FFQ has been validated using diet records with correlations of 0.6 for coffee and 0.8 for tea (A Wolk, unpublished data, 1992). Finally, residual or unmeasured confounding by lifestyle or other dietary factors that are associated with coffee and/or tea consumption is a possibility. However, we adjusted for a number of potential confounders including smoking status, and the association did not materially change.

To our knowledge, this is the largest study among women with breast cancer to examine the association between coffee consumption and mortality, and the first study to examine the association with primarily black tea and caffeine. With 973 total deaths, including 394 breast cancer deaths, we had the ability to examine breast cancer-specific mortality as well as how the associations 
Table 2 Hazard ratios (HR) and 95\% confidence intervals (95\% Cls) of breast cancer death by coffee intake among 3234 invasive breast cancer cases in the Swedish Mammography Cohort

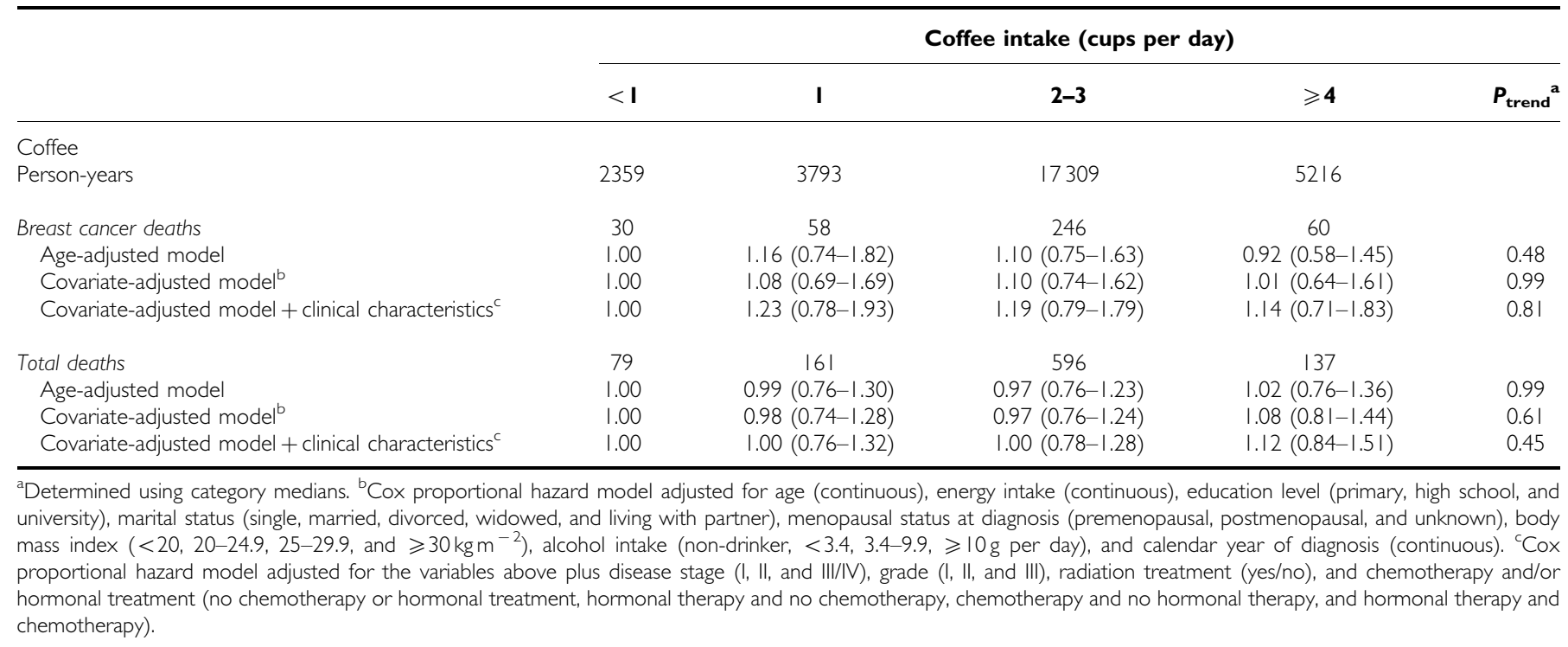

Table 3 Hazard ratios (HR) and 95\% confidence intervals (95\% Cls) of breast cancer death by tea intake among 3234 invasive breast cancer cases in the Swedish Mammography Cohort

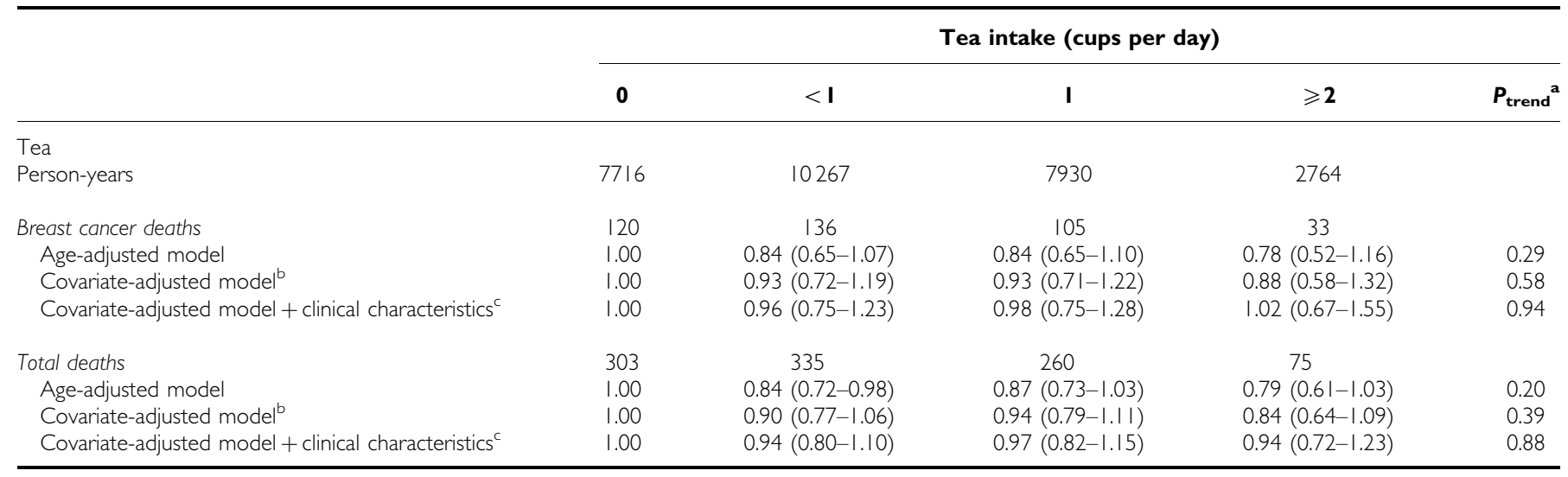

${ }^{a}$ Determined using category medians. ${ }^{b}$ Cox proportional hazard model adjusted for age (continuous), energy intake (continuous), education level (primary, high school, and university), marital status (single, married, divorced, widowed, and living with partner), menopausal status at diagnosis (premenopausal, postmenopausal, and unknown), body mass index $\left(<20,20-24.9,25-29.9, \geqslant 30 \mathrm{~kg} \mathrm{~m}^{-2}\right)$, alcohol intake (non-drinker, $<3.4,3.4-9.9$, and $\geqslant 10 \mathrm{~g}$ per d), and calendar year of diagnosis (continuous). ${ }^{\circ} \mathrm{Cox}$ proportional hazard model adjusted for the variables above plus disease stage (I, II, and III/IV), grade (I, II, and III), radiation treatment (yes/no), and chemotherapy and/or hormonal treatment (no chemotherapy or hormonal treatment, hormonal therapy and no chemotherapy, chemotherapy and no hormonal therapy, and hormonal therapy and chemotherapy).

differed by tumour hormone receptor status. We had $80 \%$ power to detect hazards ratios of 1.38 . We also have complete follow-up of all cases, a long follow-up period, detailed information on diet, and data on many important covariates, including clinical and lifestyle characteristics.

In conclusion, among a population of Swedish women with high coffee consumption, we did not observe an association between coffee, black tea, or caffeine consumption and breast cancerspecific survival or overall survival.

\section{REFERENCES}

Bhoo Pathy N, Peeters P, van Gils C, Beulens J, van der Graaf Y, Bueno-de-Mesquita B, Bulgiba A, Uiterwaal C (2010) Coffee and tea intake and risk of breast cancer. Breast Cancer Res Treat 121(2): 461-467

Bissonauth V, Shatenstein B, Fafard E, Maugard C, Robidoux A, Narod S, Ghadirian P (2009) Risk of breast cancer among French-Canadian

\section{ACKNOWLEDGEMENTS}

This work was supported by the Swedish Cancer Foundation, the Swedish Research Council/Committee for Infrastructure, the Swedish Foundation for International Cooperation in Research and Higher Education, and the Regional Research Fund Uppsala-Örebro Region.

Supplementary Information accompanies the paper on British Journal of Cancer website (http://www.nature.com/bjc)

women, noncarriers of more frequent BRCA1/2 mutations and consumption of total energy, coffee, and alcohol. Breast J 15: S63-S71 Boggs D, Palmer J, Stampfer M, Spiegelman D, Adams-Campbell L, Rosenberg L (2010) Tea and coffee intake in relation to risk of breast cancer in the Black Women's Health Study. Cancer Causes Control 21(11): 1941-1948 
Fagherazzi G, Touillaud MS, Boutron-Ruault M-C, Clavel-Chapelon F, Romieu I (2011) No association between coffee, tea or caffeine consumption and breast cancer risk in a prospective cohort study. Public Health Nutr 14(07): 1315-1320

Ferrini RL, Barrett-Connor E (1996) Caffeine intake and endogenous sex steroid levels in postmenopausal women. The Rancho Bernardo Study. Am J Epidemiol 144(7): 642-644

Gierach GL, Freedman ND, Andaya A, Hollenbeck AR, Park Y, Schatzkin A, Brinton LA (2011) Coffee intake and breast cancer risk in the NIH-AARP diet and health study cohort. Int J Cancer 131(2): 452-460

Holmes M, Chen W, Feskanich D, Kroenke C, Colditz G (2005) Physical activity and survival after breast cancer diagnosis. JAMA 293(20): 2479-2486

Ishitani K, Lin J, Manson JE, Buring JE, Zhang SM (2008) Caffeine consumption and the risk of breast cancer in a large prospective cohort of women. Arch Intern Med 168(18): 2022-2031

Kuruto-Niwa R, Inoue S, Ogawa S, Muramatsu M, Nozawa R (2000) Effects of tea catechins on the ere-regulated estrogenic activity. J Agric Food Chem 48(12): 6355-6361

Larsson S, Bergkvist L, Wolk A (2009a) Coffee and black tea consumption and risk of breast cancer by estrogen and progesterone receptor status in a Swedish cohort. Cancer Causes Control 20(10): 2039-2044

Larsson SC, Bergkvist L, Wolk A (2009b) Long-term meat intake and risk of breast cancer by oestrogen and progesterone receptor status in a cohort of Swedish women. Eur J Cancer 45(17): 3042-3046

Lee WJ, Zhu BT (2006) Inhibition of DNA methylation by caffeic acid and chlorogenic acid, two common catechol-containing coffee polyphenols. Carcinogenesis 27(2): 269-277

Li J, Seibold P, Chang-Claude J, Flesch-Janys D, Liu J, Czene K, Humphreys K, Hall P (2011) Coffee consumption modifies risk of estrogen-receptor negative breast cancer. Breast Cancer Res 13(3): R49

Mattsson B, Wallgren A (1984) Completeness of the Swedish Cancer Register. Non-notified cancer cases recorded on death certificates in 1978. Acta Radiol Oncol 23: 305-313

Maunsell E, Drolet M, Brisson J, Robert J, Deschênes L (2002) Dietary change after breast cancer: extent, predictors, and relation with psychological distress. J Clin Oncol 20(4): 1017-1025

Nagata C, Kabuto M, Shimizu H (1998) Association of coffee, green tea, and caffeine intakes with serum concentrations of estradiol and sex hormone-binding globulin in premenopausal Japanese women Nutr Cancer 30(1): 21-24

Nilsson L, Johansson I, Lenner P, Lindahl B, Van Guelpen B (2010) Consumption of filtered and boiled coffee and the risk of incident cancer: a prospective cohort study. Cancer Causes Control 21(10): 1533-1544

Petrek J, Sandberg W, Cole M, Silberman M, Collins D (1985) The inhibitory effect of caffeine on hormone-induced rat breast cancer. Cancer 56(8): 1977-1981

Pozner J, Papatestas A, Fagerstrom R, Schwartz I, Saevitz J, Feinberg M, Aufses AJ (1986) Association of tumor differentiation with caffeine and coffee intake in women with breast cancer. Surgery 100(3): 482-488

Salminen EK, Lagstrom HK (2000) Does breast cancer change patients' dietary habits? Eur J Clin Nutr 54(11): 844-848

Stocks P (1970) Cancer mortality in relation to national consumption of cigarettes, solid fuel, tea and coffee. Br I Cancer 24(2): 215-225

Sugiyama K, Kuriyama S, Akhter M, Kakizaki M, Nakaya N, OhmoriMatsuda K, Shimazu T, Nagai M, Sugawara Y, Hozawa A, Fukao A, Tsuji I (2010) Coffee consumption and mortality due to all causes, cardiovascular disease, and cancer in Japanese women. J Nutr 140(5): 1007-1013

Tang N, Zhou B, Wang B, Yu R (2009) Coffee consumption and risk of breast cancer: a metaanalysis. Am J Obstet Gynecol 200(3): 290.e291-290.e299

Trevisanato SI, Kim YI (2000) Tea and health. Nutr Rev 58(1): 1-10

VanderPloeg L, Wolfrom D, Rao A, Braselton W, Welsch C (1992) Caffeine, theophylline, theobromine, and developmental growth of the mouse mammary gland. J Environ Pathol Toxicol Oncol 11(3): 177-189

Velentzis L, Keshtgar M, Woodside J, Leathem A, Titcomb A, Perkins K, Mazurowska M, Anderson V, Wardell K, Cantwell M (2011) Significant changes in dietary intake and supplement use after breast cancer diagnosis in a UK multicentre study. Breast Cancer Res Treat 128(2): 473-482

Welsch CW, Scieszka KM, Senn ER, Dehoog JV (1983) Caffeine (1,3,7trimethylxanthine), a temperate promoter of DMBA-induced rat mammary gland carcinogenesis. Int J Cancer 32(4): 479-484

Wolk A, Larsson SC, Johansson J-E, Ekman P (2006) Long-term fatty fish consumption and renal cell carcinoma incidence in women. JAMA 296(11): 1371-1376

Wu AH, Arakawa K, Stanczyk FZ, Van Den Berg D, Koh W-P, Yu MC (2005) Tea and circulating estrogen levels in postmenopausal Chinese women in Singapore. Carcinogenesis 26(5): 976-980

This work is published under the standard license to publish agreement. After 12 months the work will become freely available and the license terms will switch to a Creative Commons Attribution-NonCommercial-Share Alike 3.0 Unported License. 